\title{
Abrasive wear behavior of PTFE for seal applications under abrasive-atmosphere sliding condition
}

\author{
Mingxue SHEN ${ }^{1,2}$, Bo LI' ${ }^{2}$, Zhinan ZHANG ${ }^{1, *}$, Longzhi ZHAO $^{2}$, Guangyao XIONG ${ }^{2, *}$ \\ ${ }^{1}$ School of Mechanical Engineering, Shanghai Jiao Tong University, Shanghai 200240, China \\ ${ }^{2}$ College of Materials Science \& Engineering, East China Jiaotong University, Nanchang 330013, China \\ Received: 07 January 2019 / Revised: 31 March 2019 / Accepted: 08 May 2019 \\ (C) The author(s) 2019.
}

\begin{abstract}
Abrasive wear is a common failure phenomenon that often limits the service life of sealing elements. Evaluation and comparison of the abrasion resistance of polytetrafluoroethylene (PTFE) were conducted using $\mathrm{Al}_{2} \mathrm{O}_{3}$ particles with sizes in the range 5 to $200 \mu \mathrm{m}$ on a pin-on-flat tribo-tester under dry reciprocating sliding conditions at room temperature. Based on the examined worn surface characteristics of both PTFE and 316L stainless steel (as a counterpart) and the analyzed coefficient of friction (COF) evolutions, the wear mechanism and particle size effect have been explored in detail. The results demonstrate that the abrasive size is the main contributing factor, which can drastically impact the wear mechanism and tribological properties of tribo-pairs. The COF exhibits different evolution characteristics (trends) for different abrasive sizes. For moderate particle sizes, the COF trends become more complicated and the most evident wear of the metallic counterpart is evident. The activity behaviors of abrasives are dominated by the particle size. Particles can becomes embedded in one of the tribo-pair materials to plough-cut the counterpart, thus causing two-body abrasive wear. The abrasives can also behave as free rolling bodies, which play the role of third body to realize three-body "PTFEabrasive-316L" abrasion. When abrasives are involved in the wear process, both the wear rate and COF of the metallic counterpart increase, but the material removal rate of the PTFE is reduced. The results obtained can offer guidelines regarding the design and protection of seals.
\end{abstract}

Keywords: PTFE; seals; three-body abrasion; wear mechanism; abrasive particle size

\section{Introduction}

Polytetrafluoroethylene (PTFE) is an effective solid lubricant that exhibits several beneficial properties, including a low friction coefficient, high melting point, and good chemical stability [1,2]. These properties make PTFE a popular tribological material for sealing applications. Since its invention in the 1930's, the tribological behaviors of PTFE and PTFE-based composites have been studied extensively by many researchers. However, the majority of existing studies mainly focus on dry friction or water-lubricated conditions without considering the presence of abrasives [3-6].
In fact, the service properties of a well-designed seal require a focus on abrasion resistance of the sealing pairs of materials [7]. Service life of sealing elements is strongly influenced by abrasive wear. It may have various causes, such as air borne dust, suspended particulates in lubricating oil, wear debris due to excessive abrasion, corrosion by-products, or a rough mating surface [8]. Therefore, it is common for PTFE to exhibit poor abrasion resistance, leading to early failure and leakage in mechanical devices, thus, its application has been limited [9]. In order to prevent wear losses of PTFE material, PTFE-based composites filled with micro/nano particles or fibers have been researched widely and have been applied in the

* Corresponding authors: Zhinan ZHANG, E-mail: zhinanz@sjtu.edu.cn; Guangyao XIONG, E-mail: xiongguangyao@163.com 
industry [9-11]. Unfortunately, there are few reports on the tribological behavior of PTFE under abrasivecontaminated sliding conditions. The wear properties of PTFE and polyimide (PI) under two different conditions with simulated sand-dust and dry sliding were compared and studied by Li and Yan [12], and the results indicated that the wear rate of PTFE was much lower under sand-dust conditions than under dry sliding conditions, and that the two types of polymers exhibited different wear behaviors under sand-dust conditions. In our previous study [13], the authors explored different damage mechanisms and debris behavior for a crylonitrile-butadiene rubber (NBR) / -stainless steel tribo-pairs with varied particle sizes. As a general property, the particle size effect exists in two-body and three-body abrasion, as well as in abrasive erosion [14, 15]. Even so, the response mechanism based on the size effect is still not fully understood [7-16], although a direct dependence of the wear mechanisms and tribological properties of polymers on the abrasive particle size was confirmed by Gomez et al. [17] and Shen et al. [18].

In this study, an ingenious pin-on-flat wear testing device simulating a sand-contaminated environment was applied to assess the abrasive wear characteristics of PTFE. The aim is to explore its tribology properties and to identify the different damage mechanisms that occur in three-body abrasion conditions, and to determine a correlation between the abrasive particle size and wear behavior. Furthermore, this study can provide fundamental insights on how to improve the design and reliability of tribo-pair sealing systems, such as those used in some types of bearings and valves, especially those which operate in sand-dust environments.

\section{Experimental materials and methods}

\subsection{Test materials}

The materials used in the tests were pure PTFE material and Grade 316L stainless steel plates. 316L stainless steels exhibits attractive properties, such as high strength and good corrosion resistance, and can be used directly as a sealing pair without requiring coating. The main properties of the tested samples are listed in Table 1. PTFE was used as the pin with a linediameter of $6 \mathrm{~mm}$ and 316L stainless steel as a counterpart was processed into a $15 \mathrm{~mm} \times 25 \mathrm{~mm}$ plates. Prior to testing, the flat specimens were polished to a surface roughness $\left(R_{\mathrm{a}}\right)$ of approximately $0.05 \mu \mathrm{m}$. Industrial grade $\mathrm{Al}_{2} \mathrm{O}_{3}$ particles, graded from 70 to 5,000 mesh, with nominal particle sizes ranging approximately from 5 to $250 \mu \mathrm{m}$, were used as abrasives. Scanning Electron Microscopy (SEM, SU8010, Hitachi, Japan) micrographs of the three types of $\mathrm{Al}_{2} \mathrm{O}_{3}$ particles are presented in Fig. 1, which illustrates the morphology of the abrasive particles.

\subsection{Test set-up and procedures}

Abrasion wear tests were implemented on a linear

Table 1 Main properties of PTFE and 316L stainless steel.

\begin{tabular}{cccccc}
\hline Material & Density $\rho\left(\mathrm{g} / \mathrm{cm}^{3}\right)$ & Hardness & Young's modulus $(\mathrm{GPa})$ & Tensile strength $\sigma_{\mathrm{b}}(\mathrm{MPa})$ & Poisson's ratio \\
\hline $316 \mathrm{~L}$ & 7.98 & $179(\mathrm{HV})$ & 205 & 580 & 0.3 \\
PTFE & 2.16 & $57.68($ Shore $D)$ & 1.28 & 30.2 & 0.46 \\
\hline
\end{tabular}

(a)

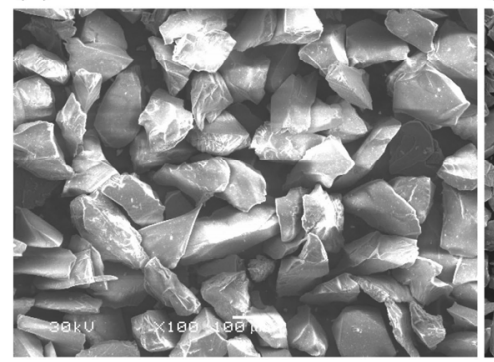

(b)

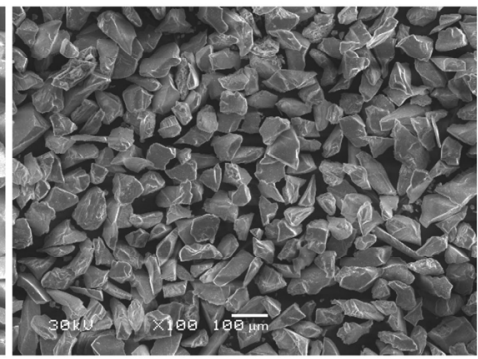

(c)

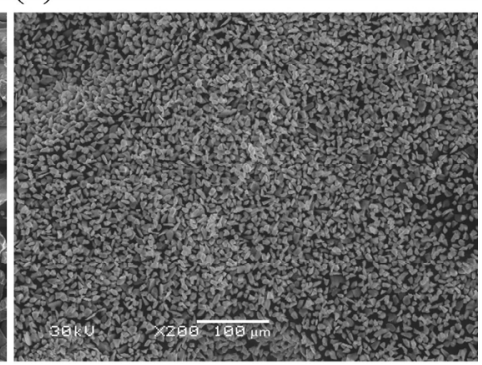

Fig. 1 SEM images of three representative $\mathrm{Al}_{2} \mathrm{O}_{3}$ particles used in the abrasion tests. Average sizes of the particles were (a) $120 \pm 20 \mu \mathrm{m}$ for 120 mesh, (b) $58 \pm 12 \mu \mathrm{m}$ for 250 mesh, and (c) $13 \pm 2 \mu \mathrm{m}$ for 1,000 mesh. 
reciprocating tribometer with a pin-on-flat contact, as shown in Fig. 2. The reciprocating sliding motion was implemented to examine the friction and wear process of the seal. The PTFE pin as the upper specimen was installed in an upper fixture which was linked to a two-dimensional force transducer. The 316L plate was mounted on a lower fixture under continuous reciprocating motion provided by the driving system. During the friction test, abrasives were fed onto the tribo-pair sample from a hopper via a slotted drum mechanism. The abrasives were introduced from both the front and back sides and the drum rotation speed was maintained constant so that the abrasives could be fed uniformly at a constant feed rate of $150 \mathrm{~g} / \mathrm{min}$. Thus, the setup guaranteed that the abrasive particles could sufficiently surround the tribo-interface and produce friction. In addition, the normal load and friction force between the tribo-pair material were measured in real-time and recorded by two-dimensional force transducers through the tester control units, as shown in Fig. 2.

A normal load $F_{\mathrm{n}}=100 \mathrm{~N}$ was applied in this study, which corresponded to a Hertz contact stress of approximately $3.54 \mathrm{MPa}$. The slip amplitude range was $20 \mathrm{~mm}$, with a constant sliding speed of $0.04 \mathrm{~m} / \mathrm{s}$. All tests were conducted at room temperature with a relative humidity of $50 \% \pm 5 \%$. All results were obtained from the average of five tests conducted for each test parameter. 316L and PTFE specimens were cleaned with acetone and distilled water in an ultrasonic bath before testing, respectively. The wear scars of both PTFE and 316L specimens were analyzed using SEM and 3D surface profilometry (Bruker, Contour GT-K, USA). In addition, the surface roughness of the worn specimens was examined by using a stylus profilometer with a $0.7 \mu \mathrm{m}$ stylus radius (3 $\mathrm{mg}$ tracking force) (Bruker, Dektak XT, USA). Furthermore, the chemical compositions on the worn surfaces were detected by using energy dispersive spectroscopy (EDX, X flash 6160, Bruker, USA) and the nano-indentation characterization of the cross-sections of wear scars was preformed a Nanomechanical test system (Anton Paar, $\mathrm{NHT}^{3}$, Austria).

\section{Results and discussion}

\subsection{Relationship between wear and abrasive sizes}

Figure 3 displays the evolution characteristics of the average friction coefficient (A-COF) for the tribo-pairs and the maximal wear depth of 316L stainless steel with increasing abrasive size. The A-COF value initially tends to ascend, then to descend gradually and finally stabilizes when the PTEF begins to rub on the 316L stainless steel. For an abrasive size of approximately $20 \mu \mathrm{m}$ (e.g., 500 mesh), the A-COF reaches its maximum value of approximately 0.32 . Moreover, after the abrasive size exceeds $120 \mu \mathrm{m}$ (e.g., 120 mesh and 70 mesh), the A-COF value is close to that under the condition where no abrasive is present. However, based on the analysis on the maximal wear depth on the surface of the 316L stainless steel, it was found that the A-COF value monotonically increased as abrasive size increased. It is worth mentioning that

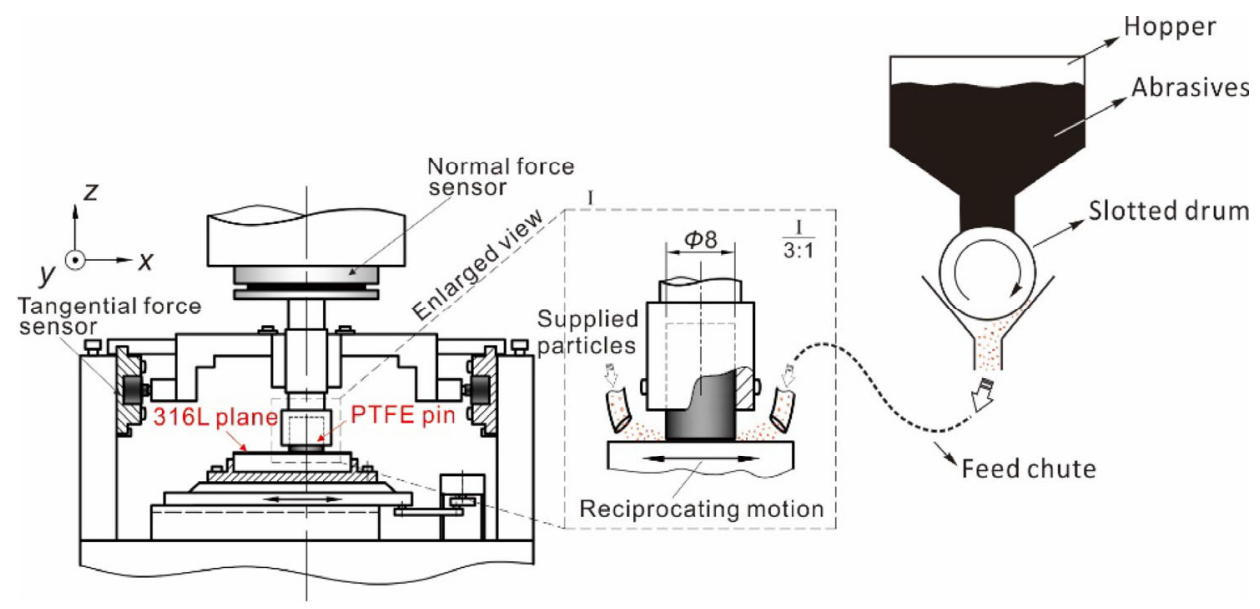

Fig. 2 Schematic of the abrasive wear test rig. 


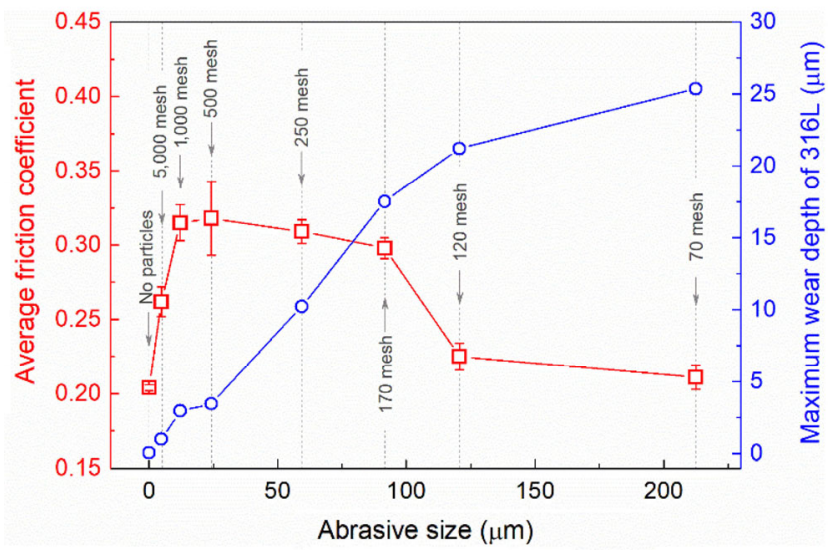

Fig. 3 Variation of average friction coefficient and maximum wear depth of $316 \mathrm{~L}$ as a function of abrasive size.

the A-COF of the tribo-pair presented a relatively low value, i.e., approximately 0.2 , under the abrasive condition created during the experiments. That is, the $\mathrm{COF}$ is higher under the abrasive wear condition than that with no abrasive, which is mainly attributed to PTFE's excellent self-lubricating property. Thus, this result is opposite to the result from the investigation on rubber three-body abrasion previously performed by our research group [13].

Further, Fig. 4 illustrates the relationship between the average wear rate and the abrasive size for both tribo-pair materials. The results indicate that it has a significant influence on the average wear rate of the tribo-pair, whether abrasives participate or not. For example, under the no-abrasive condition, friction can induce the highest wear rate for the PTFE material but almost no wear is observed for 316L stainless steel. In fact, this high wear rate of the PTFE has been attributed to its distinctive structure and to the easy removal of the transfer film formed on the counterpart surface under periodic reciprocating sliding, as reported by Amrishraj et al. and Toumi et al. [2, 4]. In contrast, the average wear rate of 316L stainless steel increases when abrasive particle are introduced. The tendency is that the average wear rate increase and decrease as the abrasive size increases. The wear extent of 316L stainless steel reaches to the maximum when the abrasive size is approximately $90 \mu \mathrm{m}$ (170 mesh). Meanwhile, for the PTFE, the wear rate maintains a small value when the size of the abrasive particles is smaller than $90 \mu \mathrm{m}$, and then the wear rate increase sharply when the abrasive size is larger than $90 \mu \mathrm{m}$.

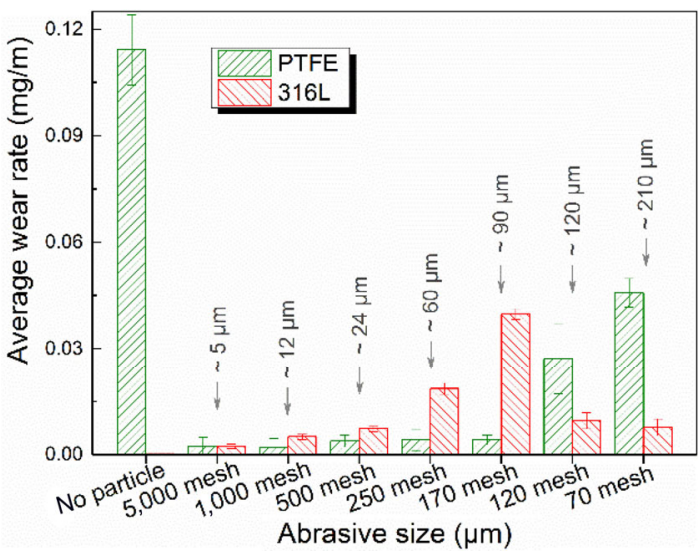

Fig. 4 Relationship between the average wear rate and the abrasive size for both tribo-pair materials: $N=5000$ cycles.

In other words, the abrasive wear behaviors of PTFE against 316L stainless steel exhibit a "particle size effect". Under the test conditions in this research, abrasive size of $90 \mu \mathrm{m}$ can be regarded as the threshold value, and this value is approximately the same critical size corresponding to metal-on-metal friction $[19,20]$.

It is significant that, for 316L stainless steel, the maximum wear depth in Fig. 3 and the average wear rate in Fig. 4 present different trends. Follow this approach, several different types of tribological characteristics can be classified and summarized, they will be discussed in detail in the following sections.

\subsection{Time-variable characteristics of friction coefficient}

As seen in Fig. 3, the COF remains at a low value $(\sim 0.2)$ in the conditions for no particles, and abrasive sizes of 70 and 120 mesh. For the purpose of discussion, we refer to particle sizes with 70 and 120 mesh as larger-size abrasive. As shown in Fig. 5, all COFs essentially remain stable during the entire wear process. More importantly, compared with the no abrasive condition, the COF (abrasive size of 70 mesh) exhibits different evolutions, which can be summarized as follows: (i) under the 70 mesh particle size and under the condition of no particles the COFs almost overlap before 3400 cycles (Stage i) and the COF values are approximately 0.201 , (ii) then the friction coefficient increases slightly to 0.211 (Stage ii). It also can be seen in Fig. 5(a) that the time-variation curve of COF for 120 mesh can be divided into two stages similar to Stage i and Stage ii. However, the COF under this 


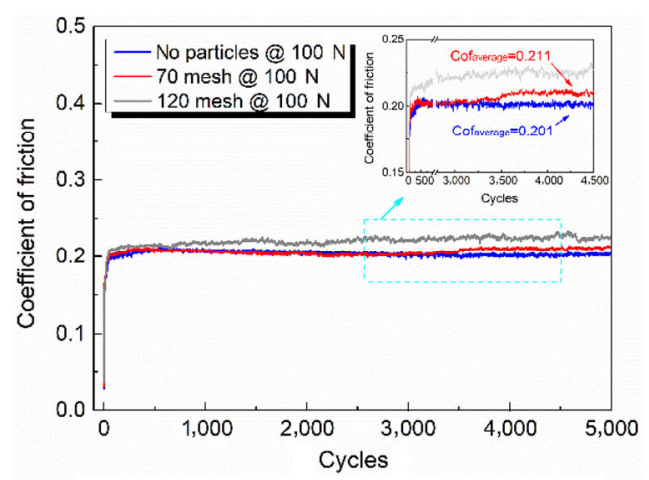

(a) No particles, 70 and 120 mesh

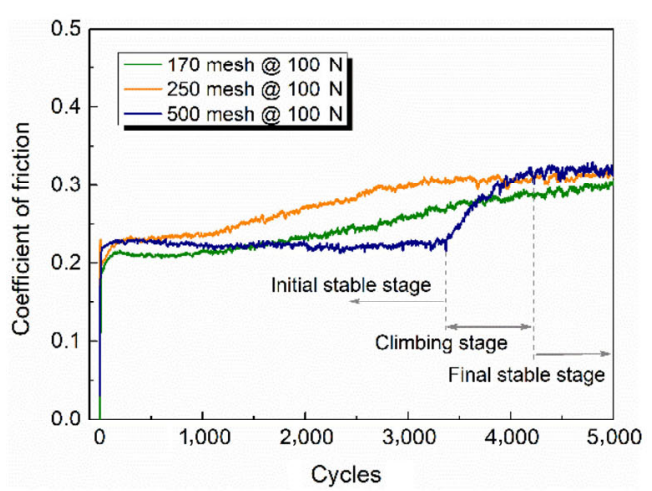

(b) 170, 250, and 500 mesh

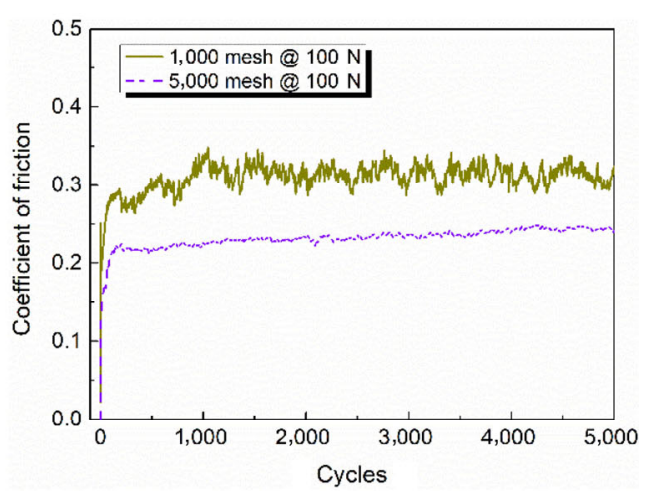

(c) 1,000 and 5,000 mesh

Fig. 5 Evolution of friction coefficients as a function of wear cycles under different abrasive sizes.

condition has already entered Stage ii after 800 cycles, so Stage ii for the 120 mesh particles appears earlier compared to that for the 70 mesh particles.

With decreasing particles sizes, particles enter the friction interface more easily during the wear process. As a result, the COF shows different characteristics under medium-size and small-size abrasive conditions. Figure 5(b) shows the evolution of the COFs as a function of cycles under medium-size abrasive conditions (corresponding to 170, 250, and 500 mesh). There are three stages apparent in the evolution of the COF: (I) initial stable stage, (II) climbing stage, and (III) final stable stage. Under the small-size abrasive condition (1,000 and 5,000 mesh particles), the COF maintains a high value (Fig. 3), and the wear rate of the PTFE is very close to the wear rate of the $316 \mathrm{~L}$ (see Fig. 4). In other words, the wear rate of the tribopair under the small-size abrasive condition is unlike the wear rate under other abrasive conditions. From Fig. 5(c) there are no periodic changes of the COF for small-size abrasives (1,000 and 5,000 mesh) over the entire friction cycle. It is also worth noting that the
COF fluctuates drastically in the 1,000 mesh condition. These phenomena are discussed and described in more detail in the following sections.

\subsection{Wear mechanism analyses}

\subsubsection{Larger abrasives}

For the two abrasive stages in Fig. 5, surface wear morphologies have been extracted for both materials of the tribo-pair. Figures 6(a) and 7(a) display the SEM images for the worn surfaces of 316L stainless steel and PFTE at 2,000 cycles, respectively. From Fig. 6(a) there is no apparent abrasive wear feature on the surface of 316L stainless steel in Stage $i$, and only slight scratch traces are visible in the locally magnified micrograph. A few instances of lamellar delamination appear on the worn surface of the PTFE and the surface is relatively smooth, as shown in Fig. 7(a). These wear morphologies are similar to those under the no-particle condition, and indicate that larger abrasive particles had difficulty entering the interface between the tribo-pair in this stage. 

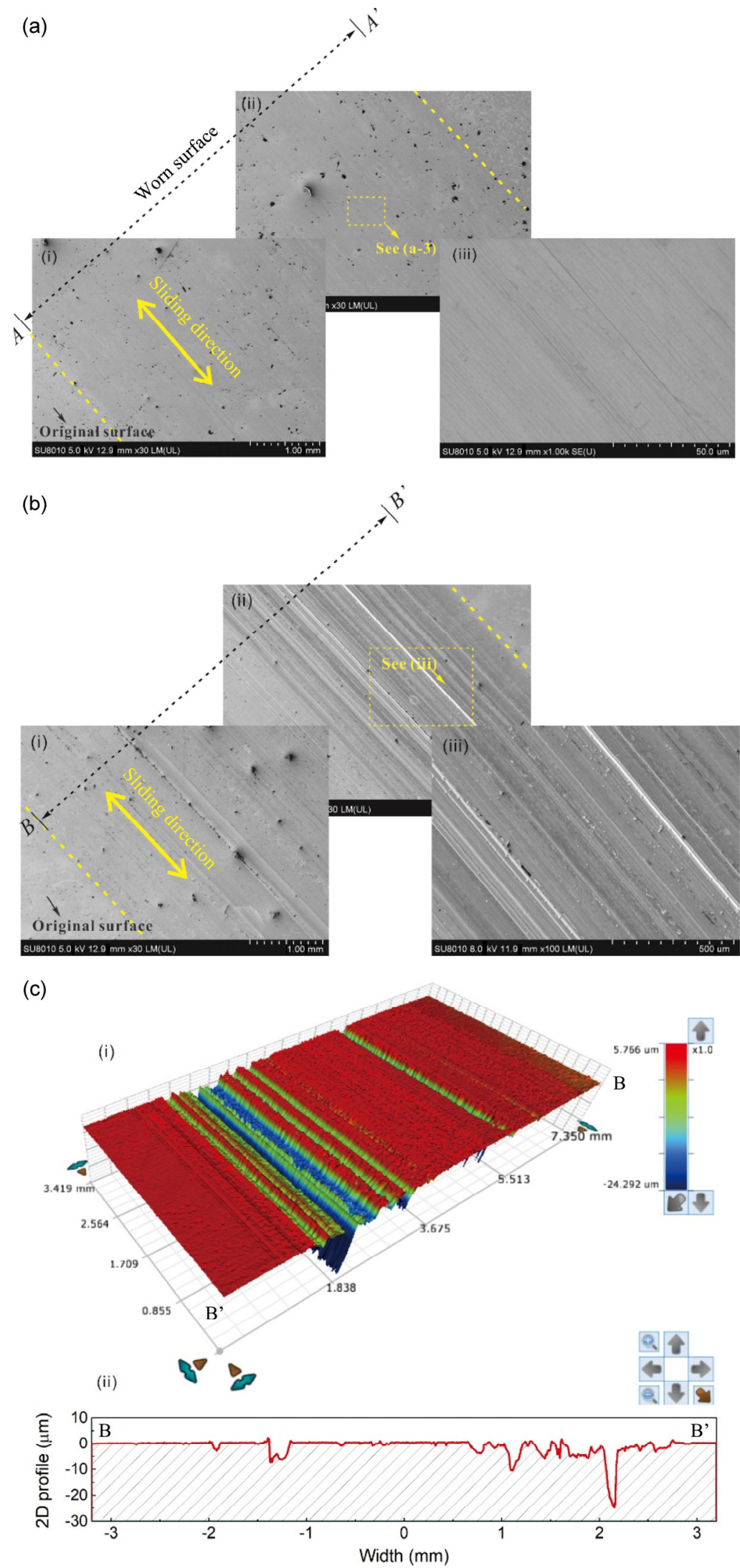

Fig. 6 SEM images ((a) and (b)), (c) 3D surface profile of the worn surface of 316L stainless steel for 70 mesh abrasives under different cycles: (a) $N=2,000$, (b) and (c) $N=5,000$. 

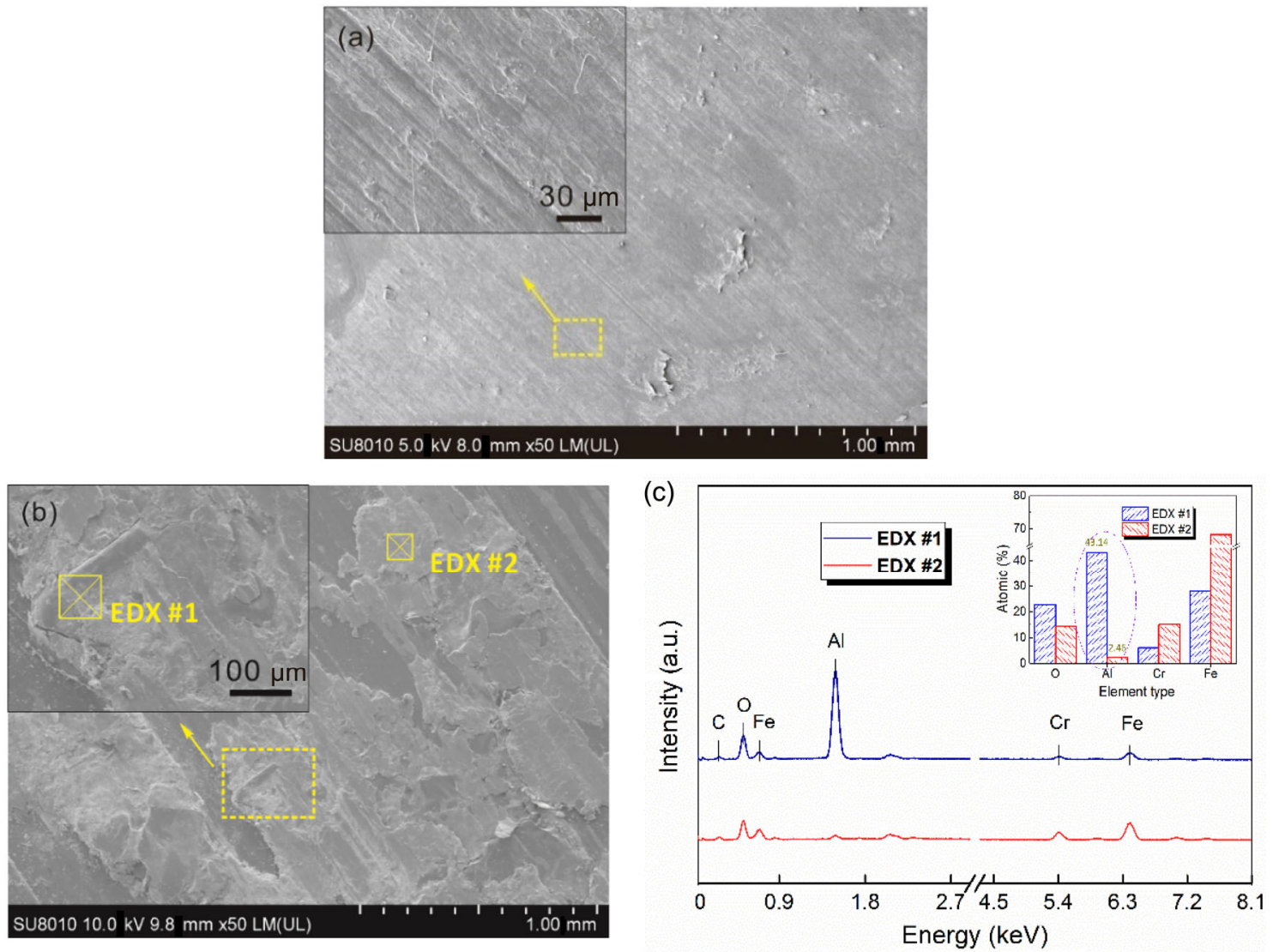

Fig. 7 SEM images ((a) and (b)), (c) EDX spectra of the worn surfaces of PTFE for 70 mesh abrasives under different cycles: (a) $N=$ 2,000, (b) and (c) $N=5,000$.

However, once Stage ii is reached, abrasive wear visibly appears on the surface of the metallic counterpart and deep ploughing is apparent on one side of the worn surface, as shown in Figs. 6(b) and 6(c). The maximum wear depth in the worn zone, as shown in Fig. 6(c), reaches $25 \mu \mathrm{m}$. Furthermore, a few $\mathrm{Al}_{2} \mathrm{O}_{3}$ abrasives particles have become embedded in the PTFE matrix according to the SEM analyses of the PTFE worn surface. The presence of $\mathrm{Al}_{2} \mathrm{O}_{3}$ particles is further confirmed by EDX detection, as shown in Fig. 7(c). Therefore, the reason for the appearance of the ploughing feature is that local embedding of abrasives in the soft PTFE matrix has led to the grinding wheel effect phenomenon on the soft PTFE surface, and the hard surface of the 316L stainless steel is cut constantly. Deep ploughing occurs in some areas, which increase local material removal. However, because the abrasive sizes are fairly large, only a few hard $\mathrm{Al}_{2} \mathrm{O}_{3}$ particle to become embedded in local areas of the PTFE surface. Therefore, for most of the worn surface area, the wear is still slight, as seen in Fig. 6(c). However, for relatively small abrasives, i.e., 120 mesh, the friction interface of the PTFE matrix is embedded earlier with abrasives, so that the COF for the 120 mesh enters into Stage ii earlier than for the 70 mesh, as shown in Fig. 5.

In summary, large-size particles (e.g., 70 and 120 mesh) have difficulty entering the friction interface; therefore, similar to the no-particle condition, the removal rate of PTFE material is high above a certain particle size (Fig. 4). Nevertheless, a few large-size particles inevitably become embedded in the PTFE matrix and intensify local cutting in the tribo-pair. This causes the slight increase of the COF and the appearance of deep ploughing on the counterpart (316L) surface.

\subsubsection{Medium-size abrasives}

Taking the 500 mesh particles as an example, there are different contributing factors corresponding to 
the three different stages in shown Fig. 5, specifically: (I) initial stable stage: actually, abrasives have already entered into the interface between the tribo-pair at the beginning of sliding process. As seen in Fig. 8(a-1), there are unrestricted $\mathrm{Al}_{2} \mathrm{O}_{3}$ particles distributed on the PTFE worn surface, which separate the tribo-pair from direct contact with each other. That is, the contact condition has transformed from "PTFE-to-316L" contact to three-body "PTFE- $\mathrm{Al}_{2} \mathrm{O}_{3}-316 \mathrm{~L}$ " abrasion. In this condition, the particles can act as rolling bodies, which effectively lowers the COF. Meanwhile, significant ploughing is apparent on the worn surface of the $316 \mathrm{~L}$ caused by abrasive wear, which also leads to severe damage of the surface, as shown in Fig. 8(a-2). (II) Climbing stage: with the implementation of reciprocating sliding, some $\mathrm{Al}_{2} \mathrm{O}_{3}$ abrasives become embedded in the soft PTFE matrix, and debris begins to accumulate, and the ploughing appearing on the $316 \mathrm{~L}$ in the previous stage is filled by the debris, as shown Figs. $8(b-1)$ and $8(b-2)$. On the other hand, with the gradual increasing of abrasives embedded in the PTFE matrix, the force that obstructed the relative movement between the tribo-pair increases, and the COF exhibits climbing trend, as shown in Fig. 8. For this reason, it can be concluded that the larger the particle size is, the longer the climbing stage will last, by comparing the time-variable curve of COF of three different abrasive sizes (170, 250, and 500 mesh, respectively).
(III) Final stable stage: As the friction continues, the worn surface of the PTFE is covered by the debris layer due to the accumulation and compaction of wear debris, as illustrated by Fig. 8(c-1). In this stage, a dynamic balance between the formation and ejection of the debris is established, so the COF enters a steady stage again. At this time, the thickness of the local wear debris layer can exceed the size of abrasive particle, and the cutting action of abrasives on the tribo-pair material diminishes. Some discontinued sticky layers were noted on the surface of the wear scars (see Fig. 8(c-2)), which are typical results when adhesive wear is present. This indicates that the main wear mechanism has transformed from abrasive wear to adhesive wear.

In general, for medium particles, the micro-cutting effect caused by high contact stress (which is perpendicular to wear surface) exists during the wear process, especially in the early stages of wear. Moreover, large numbers of abrasives have more opportunities to participate in the friction and wear processes. Therefore, the 316L stainless steel exhibits a high material removal rate (see Fig. 4) and the 2D surface profile of the wear scar present a near " $W$ "-type on both sides, as observed in Fig. 9(b).

\subsubsection{Small-size abrasives}

Abrasives can enter the friction interface more easily

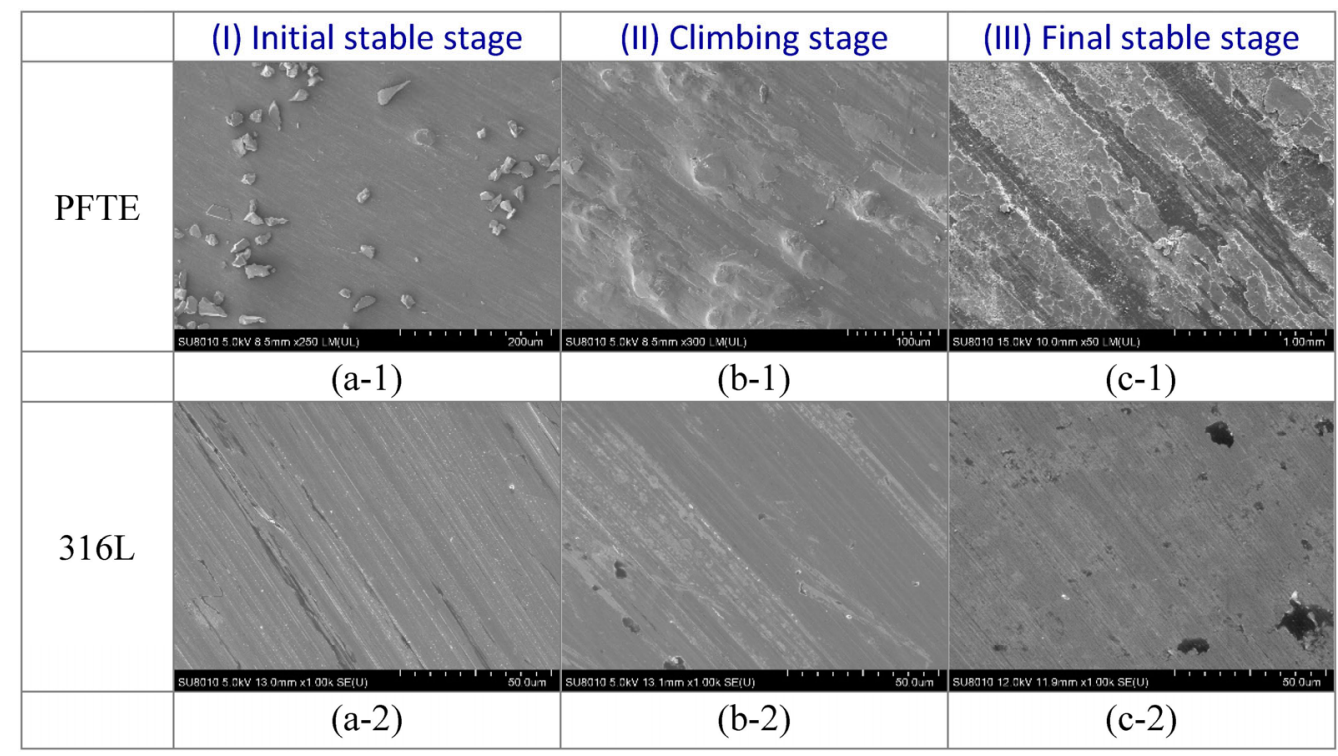

Fig. 8 SEM images of the worn surfaces of PTFE and its counterpart 316L under medium-size abrasives (i.e., 500 mesh). (a-1) and (a-2): $N=1,000,(\mathrm{~b}-1)$ and (b-2): $N=4,000,(\mathrm{c}-1)$ and (c-2): $N=5,000$. 
when the abrasive size is approximately $10 \mu \mathrm{m}$ or less (i.e., small-size abrasives), and the state of three-body abrasion caused by PTFE-abrasive-316L can be sustained during the entire wear process. That results is that the COF always maintains a stable value. According to SEM detection at high magnification, the worn surface of the 316L stainless steel is strewn with microcutting ploughs and there are some black blocky areas, which represent sticky layers, see Fig. 10(a-1). Shallow scratches and grooves are present across the entire worn surface of 316L (see the 3D profile in Fig. 10(a-3)). As shown in Fig. 10(a-2), the EDX surface scan analysis shows that many $\mathrm{Al}$ elements are distributed in the black regions, indicating that $\mathrm{Al}_{2} \mathrm{O}_{3}$ particles have become embedded in the 316L stainless steel surface. However, this phenomenon never appeared on the PTFE worn surface. This may be because the strength of PTFE is much lower than the strength of $316 \mathrm{~L}$ stainless steel and the abrasives can not embed into the PTFE firmly when the particle size is small, so the micro-cutting effect of the abrasive on the 316L stainless steel is weak. Thus, the abrasive rate of $316 \mathrm{~L}$ stainless steel under this condition is lower than that under other abrasive conditions (Fig. 4). In contrast, the
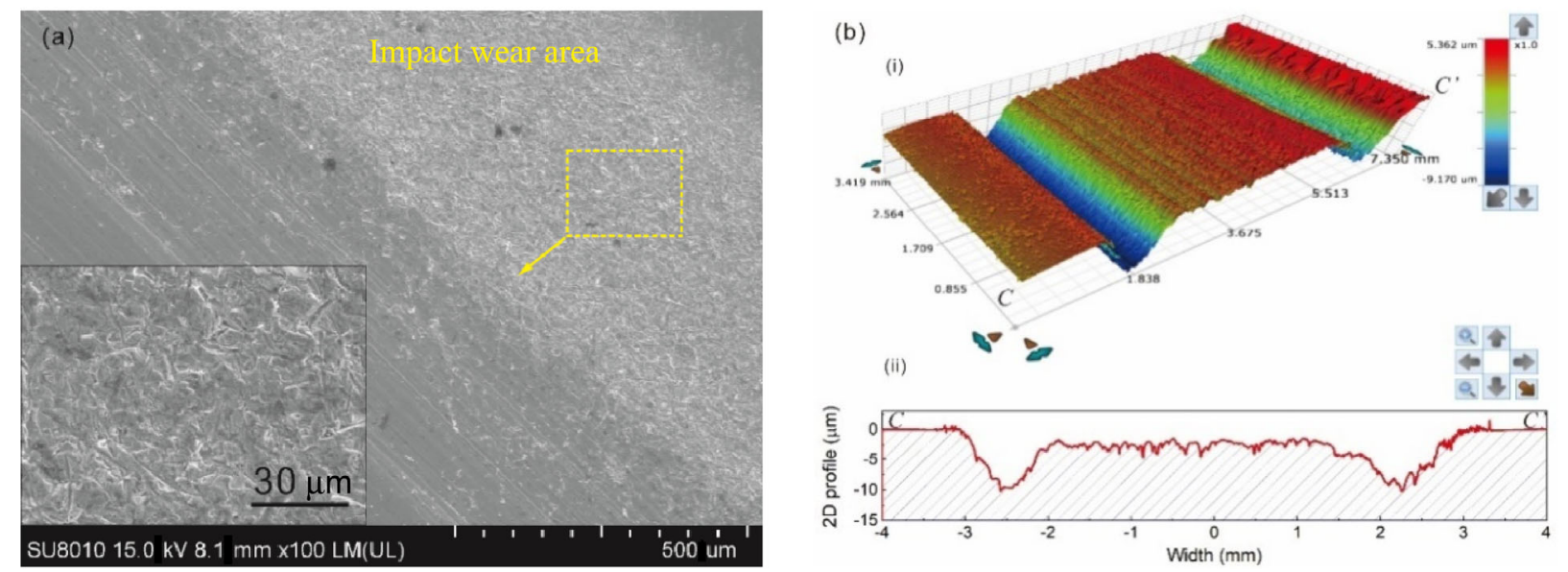

Fig. 9 3D profiles of the wear scars when the PTFE rubs on the metallic counter-part under medium-size particle abrasive conditions: 250 mesh, $N=5,000$

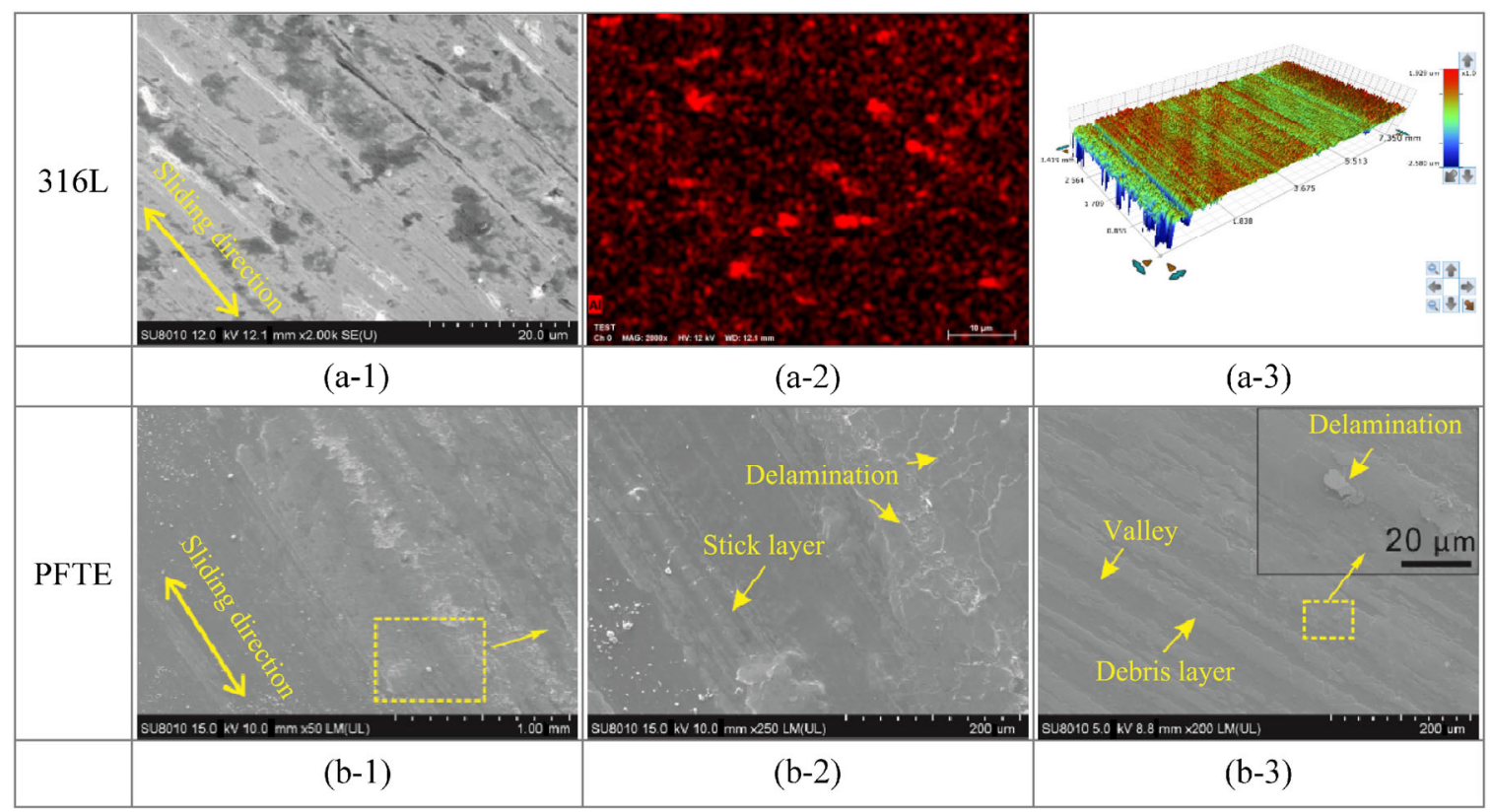

Fig. 10 SEM images, Al element distributions, and 3D profiles of the wear scars of the tribo-pair when the PFTE rubs on the metallic counter-part under two different abrasive conditions. (a-1) to (a-3), (b-1), and (b-2): 5,000 mesh, (b-3): 1,000 mesh. 
abrasives can embed into the substrate of 316L stainless steel, resulting in the accumulated debris being distributed along the sliding direction instrips or bands, which can be observed on the worn surface of the PTFE and the abrasive layer-like strips or bands are accompanied by delamination features, as shown in Figs. 10(b-1) and 10(b-2). Similar features can also be observed for 1,000 mesh particles (Fig. 10(b-3)). It should be pointed out that the valley between the thick debris layers likely provides a channel for the free abrasives passing through the wear interface probably, and the free abrasives which enter into the friction interface at this moment cannot produce the micro-cutting effect with high contact stress. Therefore, the removal rates are similar for both tribo-pair materials.

\subsection{Further insights into the wear process}

The following conclusions can also be drawn according to the test results. When the PTFE rubs on the surface of 316L stainless steel under various abrasive sizes, the worn surface morphologies of the tribo-pair exhibits significantly different damage characteristics, which could be termed as the "Particle size effect". To seek further insight into the wear process, schematic diagrams of three typical wear mechanisms are described, as shown in Fig. 11.

It is difficult for larger-size particles to enter into the friction interface. Therefore, most of the area of the contact interface shows no evident damage, and the friction and wear between the tribo-pair are similar to those of the no-abrasive condition. However, once abrasives enter the contact region, they will embed into the soft PTFE and begin to plough and loosen the surface of the hard metallic counterpart (Type I in Fig. 11). This is the reason why the nano-indentation hardness on the surface (e.g. $l \leq 10 \mu \mathrm{m})$ and subsurface (e.g. $l \geq 20 \mu \mathrm{m}$ ) is lower than that of the substrate, as shown in Fig. 12.

For the medium-size abrasives, many particles have entered the friction interface at the initial stage of sliding and the abrasive particles between the tribopair play a role as free particles. Under the continuous rolling action of the particles, the metal matching surface appears work hardening phenomenon, and the hardness of the surface layer material is significantly

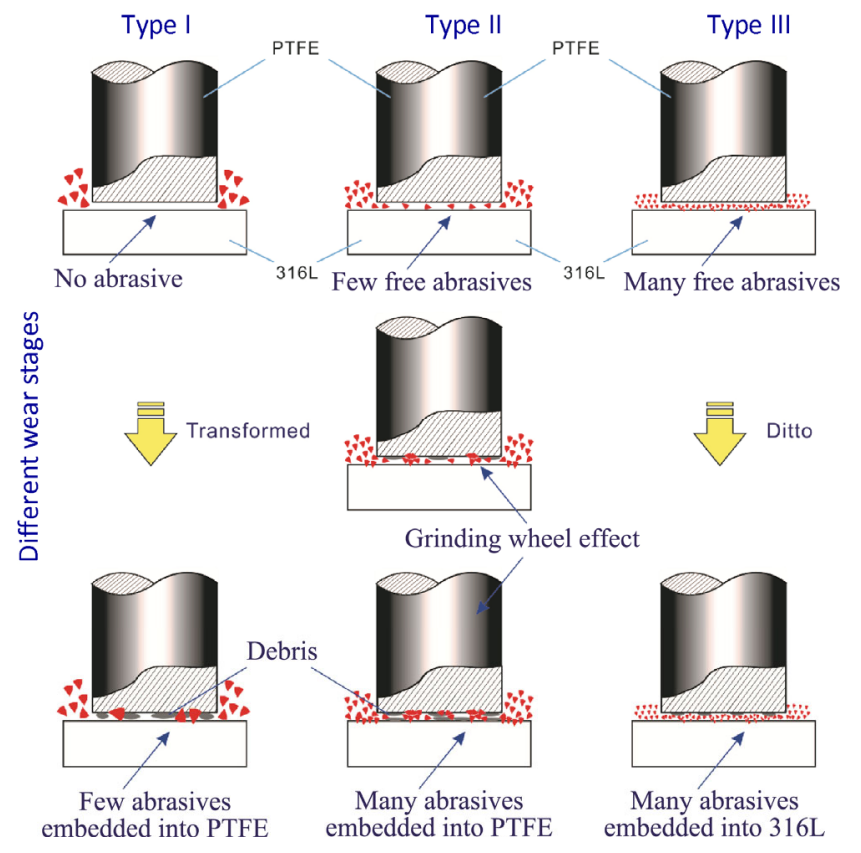

Fig. 11 Schematic sketches of the typical wear mechanisms when the PTFE is rubbing on the metallic counterpart for different abrasive sizes.

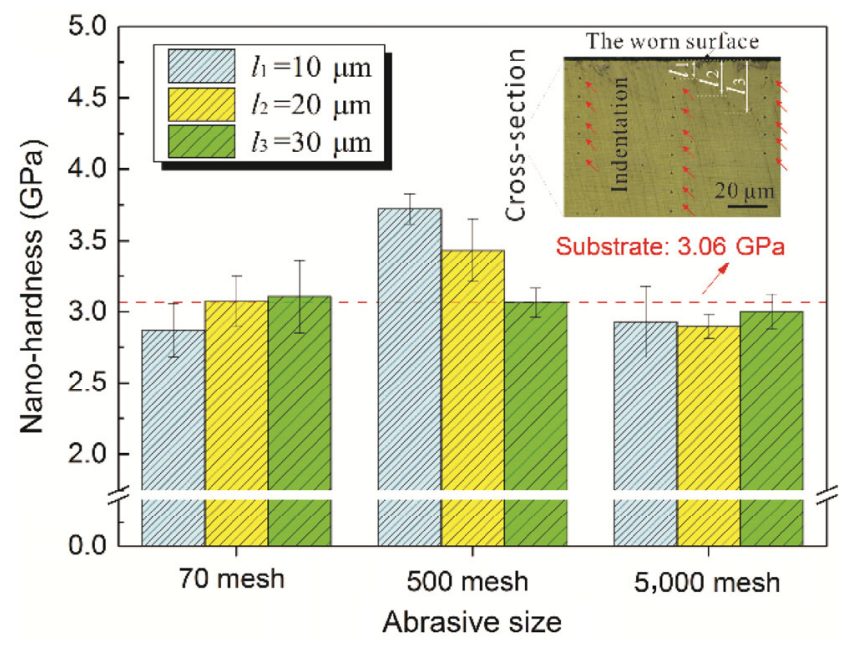

Fig. 12 Nano-hardness of different depths of the wear scar section for different particle sizes (70, 500, and 5,000 mesh).

higher than that of the substrate, as shown in Fig. 12. Thus, the load-carrying capacity is increased and the wear life is prolonged. However, as the sliding process proceeds, free particles can become firmly embedded in the PTFE matrix and gradually become abrasives, resulting in the "grinding wheel effect" which causes rapid wear loss in the metallic counterpart, although the work hardening phenomenon continues to exist on the surface of the counterpart. Meanwhile, the wear debris is constantly accumulated in the contact 
region as the cycles continue and covers the embedded abrasive, gradually resulting in the three-body (PTFE-debris-metal) abrasion state. But at the edges of the contact region, abrasives can effectively perform micro-cutting, which results in low-lying valleys formed on both sides of the contact region (Type II in Fig. 11).

However, small-size abrasives cannot embed into the PTFE firmly, so they only act as free third bodies, resulting in the three-body abrasion phenomenon (Type III in Fig. 11), so the damage is slight on both tribo-pair materials. Owing to the accumulation of abrasive particles embedded in the 316L stainless steel surface, the steel surface hardness is lower than the hardness of the substrate, as shown in Fig. 12.

\section{Conclusions}

This work investigates the influence of abrasive particle size on the friction and wear characteristics of PTFE for seal applications. Abrasive-atmosphere reciprocating sliding wear tests of PTFE pins rubbing against 316L stainless steel discs were carried out, and the mechanical wear and particle size effects have been explored in detail. The main conclusions can be summarized as follows:

(1) When hard particles were involved in the abrasive wear process, the COF of the PTFE rubbing on 316L stainless steel increased and the removal rate of the metallic counterpart greatly increased, but the abrasive rate of the PTFE was reduced owing to the presence of abrasives. As the counterpart in the tribo-pair, 316L stainless steel exhibited the highest wear rate under the medium-size abrasive particle condition. However, the maximum abrasive depth increased with increasing abrasive size.

(2) The COF exhibited noticeably different evolution rules for different abrasive sizes. For large-size abrasive particle, the COF of PTFE rubbing against 316L stainless steel was similar to that when no particle are present. However, a few particles inevitably embedded in the PTFE matrix, which resulted in a slight increase of the COF. For medium-size abrasives, the COF trend could be divided into three stages (initial stable stage, climbing stage, and final stable stage). For small-size abrasives, the three-body abrasion state could be sustained over the entire wear process and the time- variable characteristics of the COF were relatively simple. The abrasives could also serve as free rolling bodies, which played a role as the third body in the "PTFE-abrasive-316L" three-body wear process.

(3) The tribo-pair materials underwent different wear mechanisms under different abrasive sizes. For large-size abrasives, the tribo-pair wear mechanisms were similar to those for no abrasive (i.e., the mechanism of removed material was mainly the lamellar delamination of PTFE). As the friction cycles continued, a few abrasives became embedded in the PTFE matrix, which resulted in the appearance of deep ploughing on the surface of the 316L stainless steel. For medium-size abrasives, many abrasive particles entered the interface between the tribo-pair at the beginning of the sliding process, which caused noticeable wear on the metallic counterpart and the work hardening effect was found to occur. Finally, the abrasive mechanism of PTFE gradually transformed from abrasive wear to adhesive wear. For small-size abrasives, the wear mechanism was primarily three-body abrasion throughout the process.

\section{Acknowledgements}

This work was supported by the National Natural Science Foundation of China (Nos. 51775503 and 51875343), the Natural Science Foundation of Zhejiang Province (No. LY17E050020), the China Postdoctoral Science Foundation (Nos. 2017M620152 and 2018T110392), and Jiangxi Natural Science Foundation of China (20171BCD40009).

Open Access This article is licensed under a Creative Commons Attribution 4.0 International Li-cense, which permits use, sharing, adaptation, distribution and reproduction in any medium or for-mat, as long as you give appropriate credit to the original author(s) and the source, provide a link to the Creative Commons licence, and indicate if changes were made.

The images or other third party material in this article are included in the article's Creative Commons licence, unless indicated otherwise in a credit line to the material. If material is not in-cluded in the article's Creative Commons licence and your intended use is not permitted by statutory regulation or exceeds the 
permitted use, you will need to obtain permission directly from the copyright holder.

To view a copy of this licence, visit http://creativecommons.org/licenses/by/4.0/.

\section{References}

[1] Makowiec M E, Blanchet T A. Improved wear resistance of nanotube- and other carbon-filled PTFE composites. Wear 374-375: 77-85 (2017)

[2] Amrishraj D, Senthilvelan T. Development of wear mechanism maps for acrylonitrile butadiene styrene hybrid composites reinforced with nano zirconia and PTFE under dry sliding condition. J Tribol 141(2): 021602 (2019)

[3] Li J B, Liu S, Yu A B, Xiang S T. Effect of laser surface texture on CuSn6 bronze sliding against PTFE material under dry friction. Tribol Int 118: 37-45 (2018)

[4] Toumi S, Fouvry S, Salvia M. Prediction of sliding speed and normal force effects on friction and wear rate evolution in a dry oscillating-fretting PTFE/Ti-6Al-4V contact. Wear 376-377: 1365-1378 (2017)

[5] Xiong D S, Qin Y K, Li J L, Wan Y, Tyagi R. Tribological properties of PTFE/laser surface textured stainless steel under starved oil lubrication. Tribol Int 82: 305-310 (2015)

[6] Golchin A, Simmons G F, Glavatskih S B. Break-away friction of PTFE materials in lubricated conditions. Tribol Int 48: 54-62 (2012)

[7] Sui H, Pohl H, Schomburg U, Upper G, Heine S. Wear and friction of PTFE seals. Wear 224(2): 175-182 (1999)

[8] Ramadan M A. Friction and wear of sand-contaminated lubricated sliding. Friction 6(4): 457-463 (2018)

[9] Xiang D H, Shan K L. Friction and wear behavior of self-lubricating and heavily loaded metal-PTFE composites. Wear 260(9-10): 1112-1118 (2006)

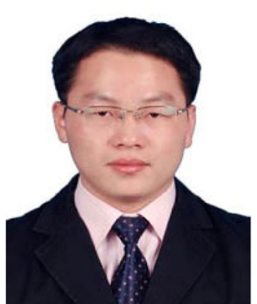

Mingxue SHEN. He received his Ph.D. degree in 2012 from Southwest Jiaotong University, Chengdu, China. After then, he was a post doctor in Shanghai Jiao Tong University. He
[10] Amrishraj D, Senthilvelan T. Dry Sliding wear behavior of ABS Composites reinforced with nano Zirconia and PTFE. Mater Today Proc 5(2): 7068-7077 (2018)

[11] Ye J, Khare H S, Burris D L. Transfer film evolution and its role in promoting ultra-low wear of a PTFE nanocomposite. Wear 297(1-2): 1095-1102 (2013)

[12] Li C X, Yan F Y. A comparative investigation of the wear behavior of PTFE and PI under dry sliding and simulated sand-dust conditions. Wear 266(7-8): 632-638 (2009)

[13] Shen M X, Zheng J P, Meng X K, Li X, Peng X D. Influence of $\mathrm{Al}_{2} \mathrm{O}_{3}$ particles on the friction and wear behaviors of nitrile rubber against $316 \mathrm{~L}$ stainless steel. $J$ Zhejiang Univ Sci A 16(2): 151-160 (2015)

[14] Coronado J J. Abrasive size effect on friction coefficient of AISI 1045 steel and 6061-T6 aluminium alloy in two-body abrasive wear. Tribol Lett 60(3): 40 (2015)

[15] de Pellegrin D V, Torrance A A, Haran E. Wear mechanisms and scale effects in two-body abrasion. Wear 266(1-2): 13-20 (2009)

[16] Zhang H J, Liu S H, Xiao H P. Sliding friction of shale rock on dry quartz sand particles. Friction 7(4): 307-315 (2019)

[17] Gomez V A O, de Macêdo M C S, Souza R M, Scandian C. Effect of abrasive particle size distribution on the wear rate and wear mode in micro-scale abrasive wear tests. Wear 328-329: 563-568 (2015)

[18] Shen M X, Dong F, Zhang Z X, Meng X K, Peng X D. Effect of abrasive size on friction and wear characteristics of nitrile butadiene rubber (NBR) in two-body abrasion. Tribol Int 103: 1-11 (2016)

[19] Tressia G, Penagos J J, Sinatora A. Effect of abrasive particle size on slurry abrasion resistance of austenitic and martensitic steels. Wear 376-377: 63-69 (2017)

[20] Jourani A, Bouvier S. Friction and wear mechanisms of $316 \mathrm{~L}$ stainless steel in dry sliding contact: Effect of abrasive particle size. Tribol Trans 58(1): 131-139 (2015)

is now working as a professor in the College of Materials Science \& Engineering, East China Jiaotong University, Nanchang, China. His major interests are tribology properties, wear behaviors, and surface engineering. 


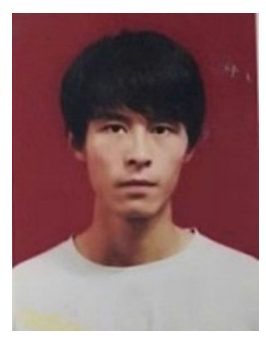

Bo LI. He received his bachelor degree in 2018 from Heilongiiang University of Science and Technology, Harbin, China. After then,

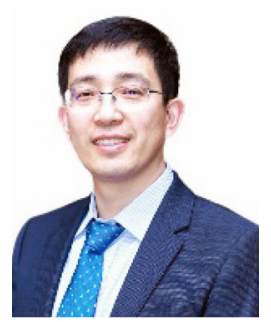

Zhinan ZHANG. He received his Ph.D. degree in 2011 from Shanghai Jiao Tong University, Shanghai, China. After that, he was a post doctor in Shanghai Jiao Tong University. He is now working as

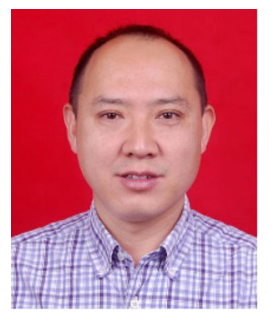

Longzhi ZHAO. He received his Ph.D. degree in 2006 from Institute of metal research, Chinese Academy of Sciences, Shenyang, China. He is

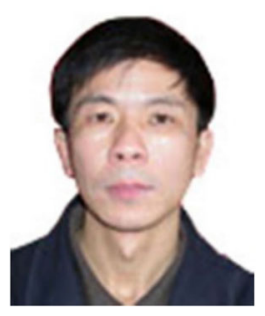

Guangyao XIONG. He received his bachelor degree in 1986 from Tianjin University, Tianjin, China. He is now working as a professor in the he was a master student in the College of Materials Science \& Engineering, East China Jiaotong University, Nanchang, China. His research interests include polymer tribology and wear behaviors.

an associate professor in the School of Mechanical Engineering, Shanghai Jiao Tong University. His research interests include computational design and analysis of tribosystems, and theory and methods of design engineering and innovation.

now working as a professor in the College of Materials Science \& Engineering, East China Jiaotong University. His research interests are laser processing technology and surface engineering.

College of Materials Science \& Engineering, East China Jiaotong University. His major interests are materials processing and surface engineering. 